\title{
Sonographic Evaluation of Cholelithiasis and Its Correlation with Normal/Fatty Liver
}

\author{
Tehreem Rehman* \\ Student, Department of Allied Health Sciences, Superior College Lahore, University Campus, 17-KM Raiwaind \\ Road, Kot Arain, Lahore, Pakistan \\ Saba Amatul Shafi \\ Student, Department of Allied Health Sciences, Superior College Lahore, University Campus, 17-KM Raiwaind \\ Road, Kot Arain, Lahore, Pakistan
}

Muhammad Usman Anwar

Student, Department of Allied Health Sciences, Superior College Lahore, University Campus, 17-KM Raiwaind Road, Kot Arain, Lahore, Pakistan

Muhammad Abbas Nazeer

Student, Department of Allied Health Sciences, Superior College Lahore, University Campus, 17-KM Raiwaind Road, Kot Arain, Lahore, Pakistan

Hasham Hafeez Hanjra

Lecturer, Department of Allied Health Sciences, Superior College Lahore, University Campus, 17-KM Raiwaind Road, Kot Arain, Lahore, Pakistan

Syed Noor ul Hassan

Lecturer, Department of Allied Health Sciences, Superior College Lahore, University Campus, 17-KM Raiwaind Road, Kot Arain, Lahore, Pakistan

Rana Muhammad Athar Azeem Shams

Masters in Medical Imaging Technology, Lecturer, Department of Allied Health Sciences, Superior College Lahore, University Campus, 17-KM Raiwaind Road, Kot Arain, Lahore, Pakistan

Rana Muhammad Bakhtawar Khan Sajawal

Masters in Mathematics, Lecturer, Department of Allied Health Sciences, Superior College Lahore, University Campus, 17-KM Raiwaind Road, Kot Arain, Lahore, Pakistan

Amna Babar

Lecturer, Superior College Lahore, University Campus, 17-KM Raiwaind Road, Kot Arain, Lahore, Pakistan

Sybil Rose

Masters in Diagnostic Ultrasound, Lecturer, Department of Allied Health Sciences, Superior College Lahore, University Campus, 17-KM Raiwaind Road, Kot Arain, Lahore, Pakistan

The research is financed by Asian Development Bank. No. 2006-A171(Sponsoring information)

\section{Abstract}

Cholelithiasis and Fatty Liver disease are usually observed to coexist. Patients who have gallstones also have Fatty Liver as both of the conditions have same associated risk factors I.e., diabetes mellitus, gender, age, obesity, metabolic syndrome, insulin resistance and high lipids level. Non-Alcoholic Fatty Liver Disease (NAFLD) is also an independent accountable risk factor for the formation of gallstones. We aimed to determine whether there is any correlation between cholelithiasis and fatty liver disease. The study was conducted in Lahore General Hospital, Lahore, Pakistan. The data was collected from March 24,2021 to May 24,2021. Informed consent was taken from all the participants. We did a cohort study comprising the data of 51 patients undergoing physical checkup. The data gathered included age, gender and whether they had normal or fatty liver. Cholelithiasis was diagnosed by confirming the presence of gallstones on abdominal ultrasonography after fasting for approximately $8 \mathrm{hrs}$. it was an observational study for the assessment of correlation between cholelithiasis and fatty liver in the participants. A Total of 51 patients with cholelithiasis were included in our study. Most of the patients were 29 to 70 years of age. The average age of the patients was $42.3 \pm 10.1$ years. Out of 51 patients, there were $60.8 \%(\mathrm{P}=31)$ females and $39.2 \%(\mathrm{P}=20)$ males. Out of 51 cholelithiasis patients there were $31.4 \%(\mathrm{P}=16)$ had Normal Liver and $68.6 \%(\mathrm{P}=35)$ were positive with Fatty liver disease. Patients with stone size 
ranges from $1.90 \mathrm{~mm}$ to $4.6 \mathrm{~mm}$ hade Grade 1 Fatty liver as shown in table-5.4, Grade 2 Fatty liver was seen in patients with stone size ranges from $4.5 \mathrm{~mm}$ to $5.8 \mathrm{~mm}$. Fatty liver Grade 3 was not observed in any included patient with cholelithiasis. Results of our study showed that both cholelithiasis and Fatty Liver disease are correlated with each other. Females are at higher risk to be affected by these with diseases. Early detection of Fatty liver in patients of cholelithiasis can help patients to prevent them further complications regarded to fatty liver and cholelithiasis and can play important role in health care of society.

Keywords: Non-Alcoholic Fatty Liver Disease, Cholelithiasis, Fatty liver, Ultrasonography.

DOI: $10.7176 / \mathrm{JHMN} / 91-07$

Publication date:July $31^{\text {st }} 2021$

\section{Introduction}

Cholelithiasis, also called gallstones, are concretions that may occur anywhere within the biliary system, most commonly within the gallbladder. They may block gallbladder or bile ducts. It is a worldwide problem and one of the common causes of surgical intervention. Gallstones are majorly composed of cholesterol and bilirubin. According to chemical composition, there are mainly three types of gallstones I.e., cholesterol stones, pigment stones and mixed stones. Cholesterol gallstones form when there is hypomobility or stasis of bile, which promotes nucleation, and hypersecretion of mucus with consequent trapping of the crystals, thus enhancing their aggregation into stones. Pigment stones are formed when there is high concentration of unconjugated bilirubin in bile in the biliary tree. The precipitates are primarily insoluble calcium bilirubinate salts. These stones may occur in patients with hemolysis or in those who have certain biliary tract infections. Mixed stones are usually composed of $20-80 \%$ cholesterol also constituting other common components i.e., bilirubin, calcium carbonate and palmitate phosphate.

Its prevalence varies in different populations. The prevalence of cholelithiasis in American adults is about $10 \%$ while the prevalence rates from $5.9 \%$ to $21.9 \%$ in western Europe. However, in Asia the prevalence ranges from $3.2 \%$ to $15.6 \%$ and in Pakistan its prevalence is $10.2 \%$. The prevalence of gallstones increases with age however the occurrence of symptomatic presentation is common in middle age, male/female ratio 1:2 respectively, obesity as its association with high cholesterol level, Type 2 diabetes, metabolic syndrome hemolysis and family history.

In about $80 \%$ of the patients, gallstones remain silent or asymptomatic while in the remaining $15-20 \%$ of the patients, gallstones are symptomatic in which patient present with biliary colic (a dull pain in the right upper quadrant i.e., right hypochondrium, discomfort or abdominal epigastric pain. Pain radiates towards right shoulder and patient may place hand behind the back and pointing thumb upwards (colin sign). Symptoms more specifically arises after having a meal enriched with fat. Other symptoms are nausea, heartburn, bloating, flinching and flatulence.

Fatty liver disease is an abnormality in which fats build up specifically triglycerides within the liver (in hepatocytes). When liver is in healthy state it is able to catabolize, synthesize and can store fatty acids and triglycerides to be used by the body as energy. Liver breaks triglycerides into Free Fatty Acid (FFA) and glycerol and store the excess or unused supplies until they are needed later. conversely, when the liver is not healthy and is not able to process the excess amounts of these molecules, the excessive or unused amount of FFAs and triglycerides are stored in hepatocytes in form of fat which results in formation of large vacuoles of fat within the cell membranes and disrupts the organelles within these cells. ${ }^{3}$ The causes of accumulation of triglycerides in hepatocytes are mainly increased supply of free fatty acids to the liver, impaired metabolism of fatty acids in hepatocytes and increased lipogenesis. Increased dietary intake or increased release of free fatty acids from adipose tissue may results in increased supply of FFAs. It might be Non-Alcoholic Fatty liver disease (NAFLD) or Alcoholic Fatty Liver Disease (AFLD). Cholelithiasis and Fatty Liver disease are commonly observed to coexist. Patients who have gallstones also have Fatty Liver as both of the conditions have same associated risk factors I.e., diabetes mellitus, gender, age, obesity, metabolic syndrome, insulin resistance and high lipids level. NAFLD is also an independent accountable risk factor for the formation of gallstones ${ }^{4}$. Globally the prevalence of NAFLD is up to $24 \%$, and this rate is increasing day by day because of the changes in life style, Junk food habits, too much food intake and lack of exercise. Insulin resistance is observed as an important feature of both cholelithiasis and fatty liver disease. ${ }^{5}$. In recent studies it is been reported that biliary cholesterol secretion may be associated with hepatic insulin resistance and hence that way promoting the formation of cholesterol gallstones formation. Hyperinsulinemia may cause super saturation of biliary cholesterol, increase secretion of hepatic cholesterol and dysmotility of gallbladder, all these conditions promote the formation of gallstones. ${ }^{6}$ A study has shown bidirectional association between cholelithiasis and fatty liver disease. ${ }^{7}$

For this research Ultrasonography is used for the evaluation of cholelithiasis and correlated fatty liver diseases, as it is non-invasive and less costly. On greyscale ultrasound cholelithiasis is seen as highly reflective echogenic foci with posterior acoustic shadowing in gallbladder lumen. Acoustic shadowing is independent of 
pathological type, composition and calcium content. By changing the patient's position, a gravity dependent movement is observed when gallstones present within the gallbladder are mobile, which is also referred as rolling stone sign. This helps in differentiating cholelithiasis from polyps that may mimic cholelithiasis ${ }^{8}$. On color doppler gallstones shows twinkling artifact and is specifically helpful in identifying small stones. Its differential diagnosis includes echogenic bile (sludge) tumefactive sludge that are non-shadowing, gallbladder polyps and gallbladder carcinoma.

Early detection of cholelithiasis in patients with fatty liver will play very vital role to reduce the complication related to cholelithiasis.

\subsection{Materials and Methods}

An observational study was performed in Lahore General Hospital, Lahore, Pakistan. Non-Probability Convenient Sampling Technique was used. The data was collected from March 24, 2021 to May 24, 2021.The rules and regulations set by the ethical committee of The Superior College, Lahore were followed while conducting the research and the rights of the research participants were respected. Written informed consent attached was taken from all the participants. We did a cohort study comprising the data of 51 patients undergoing physical checkup. The data gathered included age, gender and whether they had normal or fatty liver. Cholelithiasis was diagnosed by confirming the presence of gallstones on abdominal ultrasonography after fasting for approximately 8 hrs. A Total of 51 patients with cholelithiasis were included in our study in which 31 were females and 20 male patients. In our study patients presenting with right hypochondriac pain or discomfort, abdominal epigastric pain and patients diagnosed with cholelithiasis were included. Patients who had P/S of cholecystectomy, polyps, sludge and tumor in gallbladder were excluded. Ultrasound machine was used for abdominal scan with transducer of frequency 2.5 to $5 \mathrm{MHz}$. Data was tabulated and analyzed with the help of statistical package for the social sciences (SPSS) 25 and Microsoft excel (2016). Data was reported using cross sectional descriptive statistics. The result of quantitative variables like age, presence of Normal/Fatty Liver, presented in mean and standard deviation. The qualitative variables like gender were reported using percentages and frequencies. Bar chart were given.

1.1.1 Results

A total of 51patients with cholelithiasis were included in our study. Most of the patients were 29 to 70 years of age as shown in table-5.1. The average age of the patients was $42.3 \pm 10.1$ years. Out of 51 patients, there were $60.8 \%(\mathrm{P}=31)$ females and $39.2 \%(\mathrm{P}=20)$ males as shown in Table-5.2. Out of 51 cholelithiasis patients there were $31.4 \%(\mathrm{P}=16)$ had Normal Liver and $68.6 \%(\mathrm{P}=35)$ were positive with Fatty liver disease as shown in table5.3. Patients with stone size ranges from $1.90 \mathrm{~mm}$ to $4.6 \mathrm{~mm}$ hade Grade 1 Fatty liver as shown in table-5.4, Grade 2 Fatty liver was seen in patients with stone size ranges from $4.5 \mathrm{~mm}$ to $5.8 \mathrm{~mm}$ as presented in table-5.5. Fatty liver Grade 3 was not observed in any included patient with cholelithiasis as can be seen in table-5.6.

1.1.2 Discussion

Qiao-Hua Qiao et al., in their study, "Nonalcoholic fatty liver was associated with asymptomatic gallstones in a Chinese population". According to this study, out of 7583 patients, gallstones were diagnosed in 919 (12.12\%) patients; however, NAFLD was observed in 542/919 (58.98\%) patients. Their study revealed that people with gallstones were more likely to have NAFLD and this correlation was more usually seen in patients who aged $<50$. Young-Kyu Kim et al., also had a research with results in which NAFLD was associated with gallstones. Their study included 39135 patients out of which there were 498 cases of gallstones which coexisted with NAFLD. In another previous study review Veeravich Jaruvongvanich et al., extracted data from 12 observational studies in which the pooled OR of NAFLD in patients with cholelithiasis was 1.55 (95\% CI 1.31-1.82). The association between NAFLD and gallstones was significantly observed in cohort studies with pooled OR 1.33 (95\% CI $1.14-1.55$, I $(2)=0 \%)$. There meta-analysis found that GD is significantly associated with NAFLD. Ankit Sharma et al., conducted a study to evaluate the prevalence of Non-Alcoholic Fatty liver disease in in patients with gallstones disease. In their study total 300 patients of sonographically diagnosed with gallbladder stones were studied for NAFLD. A significant number of patients $63 \%(\mathrm{P}=189)$ showed NAFLD. However, $37 \%(\mathrm{P}=111)$ patients did not have significant steatosis. Their research showed high prevalence of NAFLD in patients with gallstones. Some studies even show bidirectional relationship between cholelithiasis and NAFLD ${ }^{5}$ Similarly, our study findings supports that there is correlation between cholelithiasis and NAFLD. In this cohort study, out of 51 patients with cholelithiasis, 20 patients were male, and 31 patients were female. There was age group between 29-70. Our study suggested that 35 patients had Fatty liver in which 13 patients were male, and 22 patients were female. Moreover, 24 patients had Grade 1 fatty liver in which 14 patients were female and 10 patients were male, where 11 patients had Fatty liver Grade 2 in which 8 patients were female and 3 patients were male, and none of them were found with Fatty liver Grade 3. It's high prevalence with cholelithiasis was suggested in some previous studies but rejected by others. ${ }^{13,14,15}$ Our study evaluates by using Ultrasonography technique. Fatty Liver was found in $68.6 \%$ patients with cholelithiasis. Previous cohort studies suggests that cholelithiasis association with NFALD was more likely observed in females ${ }^{13}$ rather than males which was also seen in our 
study as females were with more ratio than males. Older age is known as one of the risk factors for cholelithiasis. ${ }^{14,15}$ In our study, age analysis shows that fatty liver was more oftently observed in patients mid aged or older ones. Moreover, our study also reveals that as the size of cholelithiasis increases the risk of developing fatty liver disease also increases, also patients with more cholelithiasis size were observed to have higher grade of fatty liver.

1.1.3 Conclusion

Results of our study showed that both cholelithiasis and Fatty Liver disease are correlated with each other. Females are at higher risk to be affected by these with diseases. Early detection of Fatty liver in patients of cholelithiasis can help patients to prevent them further complications regarded to fatty liver and cholelithiasis and can play important role in health care of society.

\section{References:}

1. Lammert F, Gurusamy K, Ko CW, Miquel JF, Méndez-Sánchez N, Portincasa P, Van Erpecum KJ, Van Laarhoven CJ, Wang DQ. Gallstones. Nature reviews Disease primers. 2016 Apr 28;2(1):1-7.

2. Alishi YA, Howaish FA, Alhamdan FA, Almalki AA, Alqahtani SA, Alharthi SA, Alanazi AS, Alsuroji AH Prevalence and risk factors for gallstones among population in Riyadh City, KSA 2017. The Egyptian Journal of Hospital Medicine. 2017 Oct 1;69(5):2384-8.

3. Kim YK, Kwon OS, Her KH. The grade of nonalcoholic fatty liver disease is an independent risk factor for gallstone disease: An observational Study. Medicine. 2019 Jul;98(27).

4. Milić S, Lulić D, Štimac D. Non-alcoholic fatty liver disease and obesity: biochemical, metabolic and clinical presentations. World J Gastroenterol. 2014 Jul 28;20(28):9330-7. doi: 10.3748/wjg.v20.i28.9330. PMID: 25071327; PMCID: PMC4110564.

5. Chang, Yoosoo, et al. \&quot;Bidirectional association between nonalcoholic fatty liver disease and gallstone disease: a cohort study.\&quot; Journal of Clinical Medicine 7.11 (2018): 458.

6. Xie Y,Newberry EP,Kennedy SM,Luo J,Davidson NO (2009) Increased susceptibility to diet-induced gallstones in liver fatty acid binding protein knockout mice. J Lipid Res 50: 977-987.

7. Lee, Yen-Chun, et al. \&quot;Moderate to severe, but not mild, nonalcoholic fatty liver disease associated with increased risk of gallstone disease.\&quot; Scandinavian journal of gastroenterology 49.8 (2014): 10011006.

8. $\mathrm{Li}, \mathrm{Xu}$, et al. \&quot;Gallstones in patients with chronic liver diseases.\&quot; BioMed research international 2017 (2017).

9. Qiao QH, Zhu WH, Yu YX, Huang FF, Chen LY. Nonalcoholic fatty liver was associated with asymptomatic gallstones in a Chinese population. Medicine. 2017 Sep;96(38).

10. Kim, Young-Kyu, Oh-Sung Kwon, and Kyu Hee Her. \&quot;The grade of nonalcoholic fatty liver disease is an independent risk factor for gallstone disease: An observational Study.\&quot; Medicine 98.27 (2019).

11. Kim, Young-Kyu, Oh-Sung Kwon, and Kyu Hee Her. \&quot;The grade of nonalcoholic fatty liver disease is an independent risk factor for gallstone disease: An observational Study.\&quot; Medicine 98.27 (2019).

12. Sharma, Ankit, et al. \&quot;Prevalence of Non-Alcoholic Fatty Liver with Gall Stones Disease in Himalayan Population.\&quot; Journal of Evolution of Medical and Dental Sciences 9.12 (2020): 932-937.

13. Liu, Jia, et al. \&quot;Non-alcoholic fatty liver disease associated with gallstones in females rather than males: a longitudinal cohort study in Chinese urban population.\&quot; BMC gastroenterology 14.1 (2014): 213.

14. Liew PL, Lee WJ, Wang W, et al. Fatty liver disease: predictors of nonalcoholic steatohepatitis and gallbladder disease in morbid obesity. Obes Surg 2008;18:847-53.

15. Festi D, Dormi A, Capodicasa S, et al. Incidence of gallstone disease in Italy: results from a multicenter, population-based Italian study (the MICOL project). World J Gastroenterol 2008;14:5282-9.

Age * H/O Cholelithiasis:

Table No 5.1: $\quad n=51$

Age (Years)

Frequency Percent

\begin{tabular}{|c|c|c|c|}
\hline \multirow[t]{6}{*}{ Valid } & 26 & 1 & 2.0 \\
\hline & 28 & 2 & 3.9 \\
\hline & 29 & 3 & 5.9 \\
\hline & 30 & 1 & 2.0 \\
\hline & 31 & 5 & 9.8 \\
\hline & 32 & 1 & 2.0 \\
\hline
\end{tabular}




\begin{tabular}{|c|c|c|}
\hline 33 & 1 & 2.0 \\
\hline 34 & 1 & 2.0 \\
\hline 35 & 1 & 2.0 \\
\hline 37 & 1 & 2.0 \\
\hline 38 & 1 & 2.0 \\
\hline 39 & 3 & 5.9 \\
\hline 40 & 2 & 3.9 \\
\hline 41 & 1 & 2.0 \\
\hline 42 & 4 & 7.8 \\
\hline 43 & 1 & 2.0 \\
\hline 45 & 2 & 3.9 \\
\hline 46 & 3 & 5.9 \\
\hline 47 & 2 & 3.9 \\
\hline 48 & 2 & 3.9 \\
\hline 49 & 1 & 2.0 \\
\hline 50 & 2 & 3.9 \\
\hline 51 & 1 & 2.0 \\
\hline 53 & 2 & 3.9 \\
\hline 56 & 1 & 2.0 \\
\hline 57 & 1 & 2.0 \\
\hline 58 & 1 & 2.0 \\
\hline 60 & 1 & 2.0 \\
\hline 62 & 1 & 2.0 \\
\hline 63 & 1 & 2.0 \\
\hline 70 & 1 & 2.0 \\
\hline Total & 51 & 100.0 \\
\hline
\end{tabular}

Table 1: This table is about descriptive statistics of age. Bar Chart

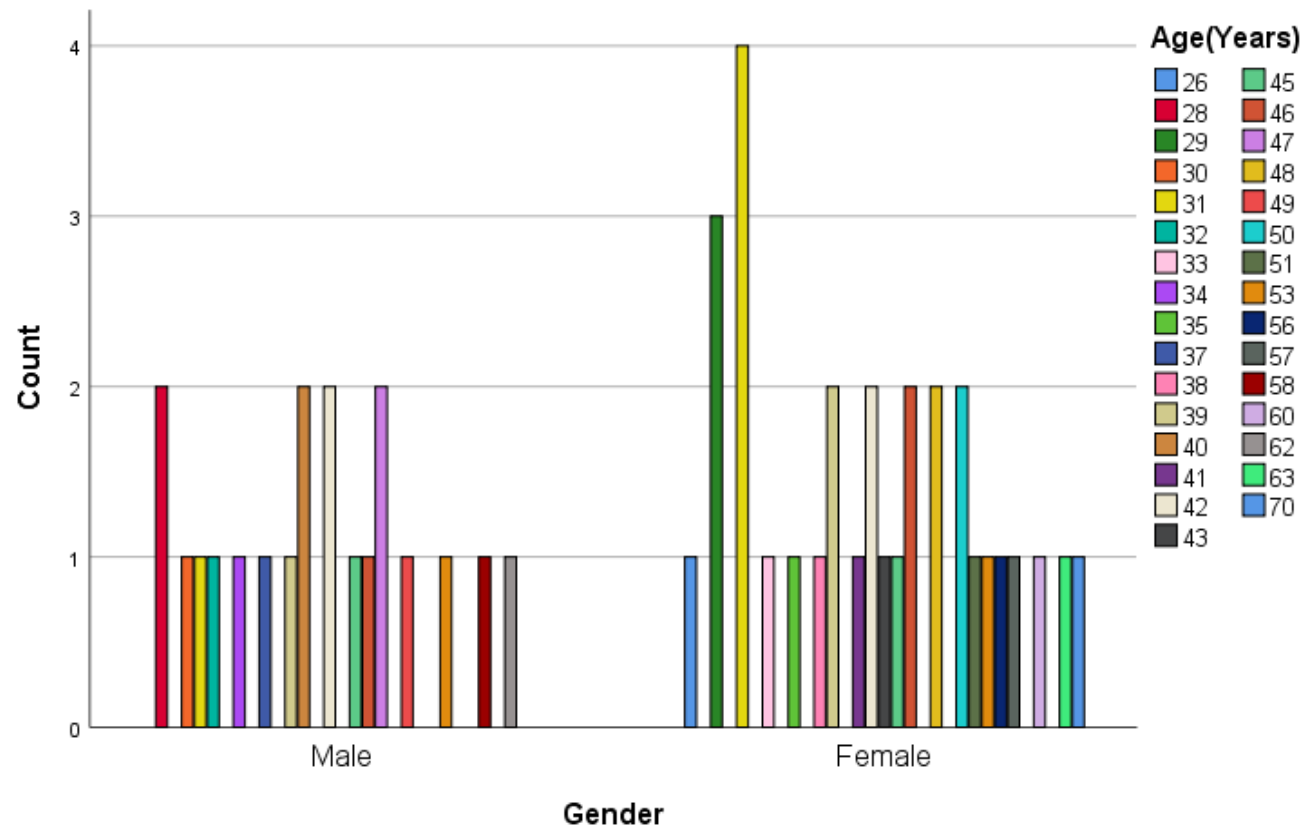

Graph 1

Gender * H/O Cholelithiasis: 
Table No 5.2: $\quad n=51$

\begin{tabular}{|llr|r|}
\multicolumn{3}{c}{ Gender } \\
& \multicolumn{2}{c}{ Frequency } & \multicolumn{1}{c}{ Percent } \\
\hline Valid & Male & 20 & 39.2 \\
\cline { 2 - 4 } & Female & 31 & 60.8 \\
\cline { 2 - 4 } & Total & 51 & 100.0 \\
\hline
\end{tabular}

Table 2: Gender-wise frequency of Cholelithiasis

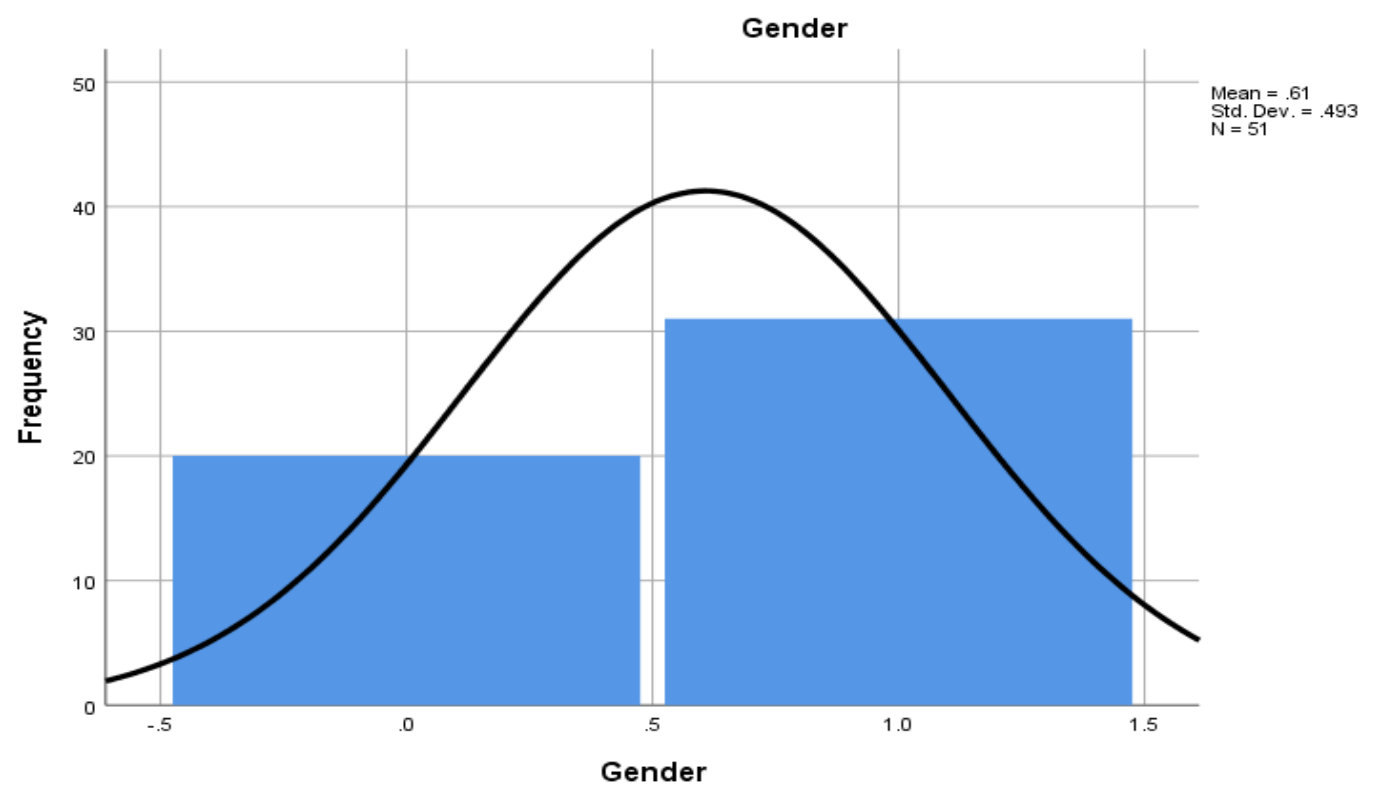

Graph 2

Frequency of Normal/Fatty Liver:

Table No 5.3: $\quad n=51$

\begin{tabular}{llr|r|}
\multicolumn{3}{c}{ Fatty liver } \\
Frequency & Percent \\
\hline Valid & NO & 16 & 31.4 \\
\cline { 2 - 4 } & YES & 35 & 68.6 \\
\cline { 2 - 4 } & Total & 51 & 100.0 \\
\hline
\end{tabular}

Table 3: Frequency of Fatty Liver 


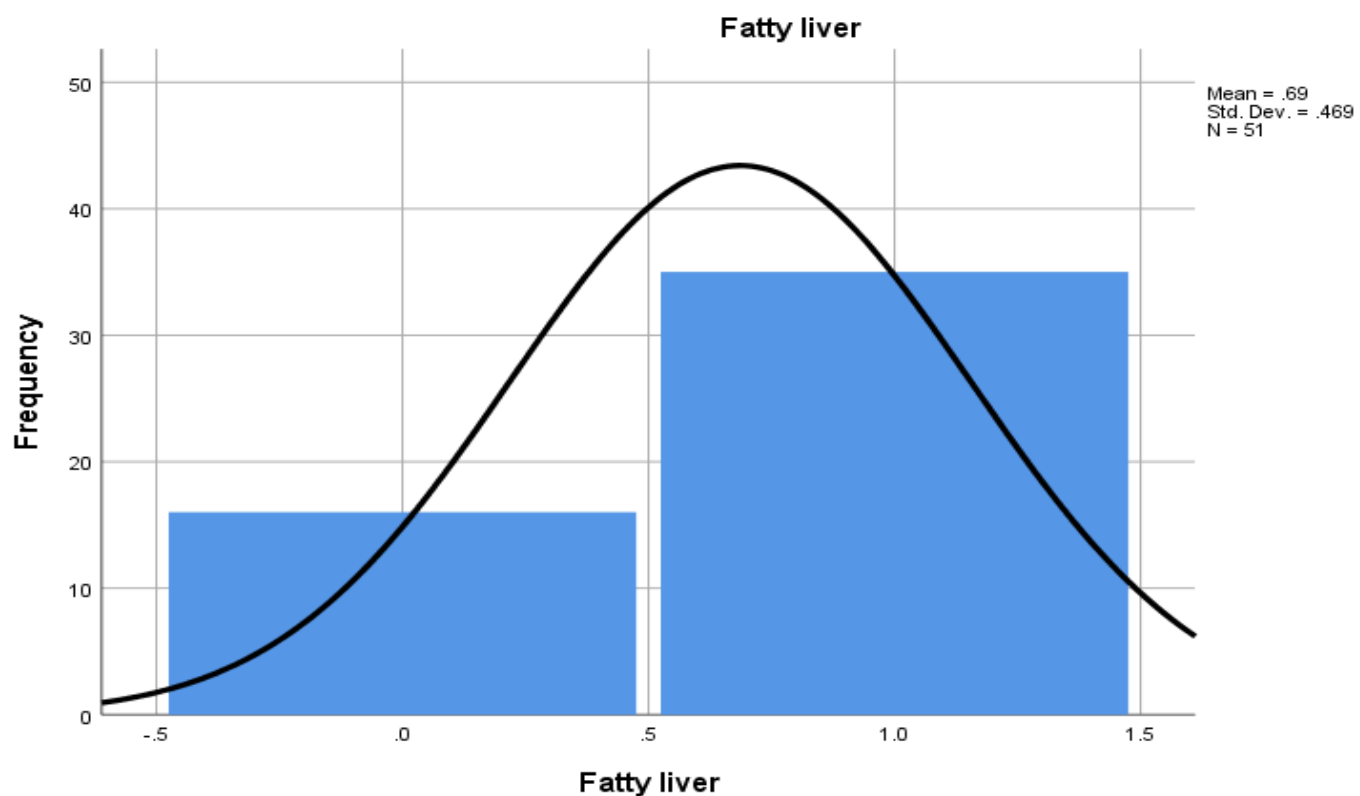

Graph 3

Cholelithiasis size Correlating with fatty Liver Grades:

Table No 5.4: $n=51$

Size $(\mathrm{mm})$ * Grade 1

Count

Grade 1

\begin{tabular}{l|r|r|r} 
& NO & YES & Total \\
\hline Size(mm) & 1 & 0 & 1 \\
\hline 1.70 & 0 & 1 & 1 \\
\hline 2.90 & 3 & 0 & 3 \\
\hline 2.10 & 3 & 1 & 4 \\
\hline 2.20 & 2 & 2 & 4 \\
\hline 2.60 & 1 & 1 & 2 \\
\hline 2.70 & 1 & 0 & 1 \\
\hline 2.80 & 1 & 0 & 1 \\
\hline 2.90 & 0 & 1 & 1 \\
\hline 3.00 & 1 & 2 & 3 \\
\hline 3.10 & 0 & 2 & 2 \\
\hline 3.20 & 1 & 3 & 4 \\
\hline 3.30 & 0 & 3 & 3 \\
\hline 3.50 & 1 & 0 & 1 \\
\hline 3.60 & 1 & 2 & 3 \\
\hline 3.70 & 0 & 2 & 2 \\
\hline 3.80 & 0 & 1 & 1 \\
\hline 3.90 & 1 & 1 & 2 \\
\hline 4.30 & 0 & 1 & 1 \\
\hline 4.50 & 1 & 0 & 1 \\
\hline 4.60 & 0 & 1 & 1 \\
\hline 5.00 & 1 & 0 & 1 \\
\hline 5.10 & 2 & 0 & 2 \\
\hline 5.20 & 1 & 0 & 1 \\
\hline & & &
\end{tabular}




\begin{tabular}{lrr|r|r}
\hline 5.50 & 1 & 0 & 1 \\
\hline 5.60 & 2 & 0 & 2 \\
\hline 5.70 & 1 & 0 & 1 \\
\cline { 2 - 5 } & 5.80 & 1 & 0 & 1 \\
\hline Total & 27 & 24 & 51 \\
\hline
\end{tabular}

Table 4: This table shows the correlation between cholelithiasis size and Fatty liver grade 1.

\section{Bar Chart}

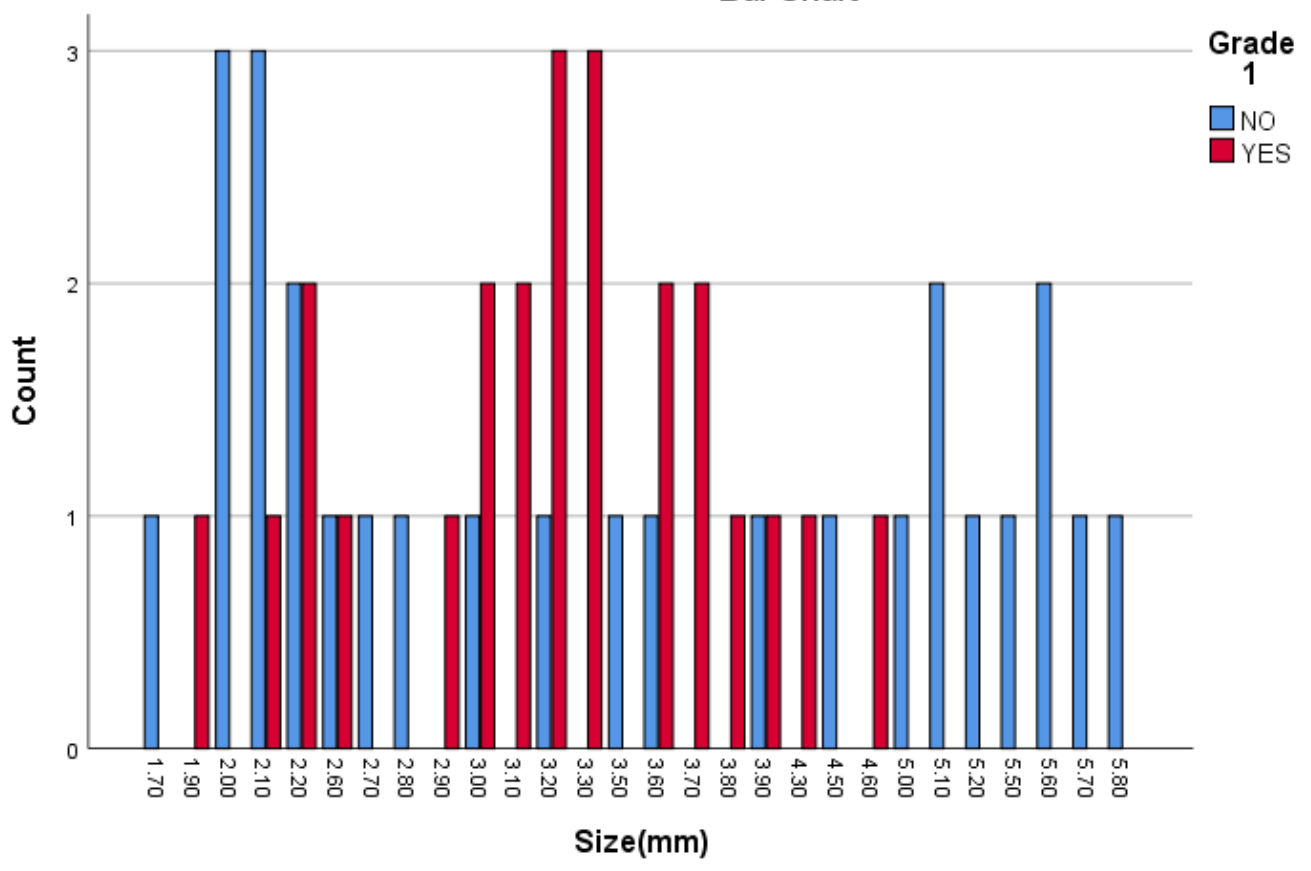

Graph 4

Table No 5.5: $\quad n=51$

\section{Size $(\mathrm{mm})$ * Grade 2}

Count

Grade 2

\begin{tabular}{|c|c|c|c|c|}
\hline $\operatorname{Size}(\mathrm{mm})$ & & NO & YES & Total \\
\hline \multirow[t]{17}{*}{$\operatorname{Size}(\mathrm{mm})$} & 1.70 & 1 & 0 & 1 \\
\hline & 1.90 & 1 & 0 & 1 \\
\hline & 2.00 & 2 & 1 & 3 \\
\hline & 2.10 & 4 & 0 & 4 \\
\hline & 2.20 & 4 & 0 & 4 \\
\hline & 2.60 & 2 & 0 & 2 \\
\hline & 2.70 & 1 & 0 & 1 \\
\hline & 2.80 & 1 & 0 & 1 \\
\hline & 2.90 & 1 & 0 & 1 \\
\hline & 3.00 & 3 & 0 & 3 \\
\hline & 3.10 & 2 & 0 & 2 \\
\hline & 3.20 & 4 & 0 & 4 \\
\hline & 3.30 & 3 & 0 & 3 \\
\hline & 3.50 & 1 & 0 & 1 \\
\hline & 3.60 & 3 & 0 & 3 \\
\hline & 3.70 & 2 & 0 & 2 \\
\hline & 3.80 & 1 & 0 & 1 \\
\hline
\end{tabular}




\begin{tabular}{lr|r|r|r}
\hline 3.90 & 2 & 0 & 2 \\
\hline 4.30 & 1 & 0 & 1 \\
\hline 4.50 & 0 & 1 & 1 \\
\hline 4.60 & 1 & 0 & 1 \\
\hline 5.00 & 0 & 1 & 1 \\
\hline 5.10 & 0 & 2 & 2 \\
\hline 5.20 & 0 & 1 & 1 \\
\hline 5.50 & 0 & 1 & 1 \\
\hline 5.60 & 0 & 2 & 2 \\
\hline 5.70 & 0 & 1 & 1 \\
\hline Total & 5.80 & 0 & 1 & 1 \\
\hline
\end{tabular}

Table 5: This table shows the correlation between cholelithiasis size and Fatty liver grade 2.

Bar Chart

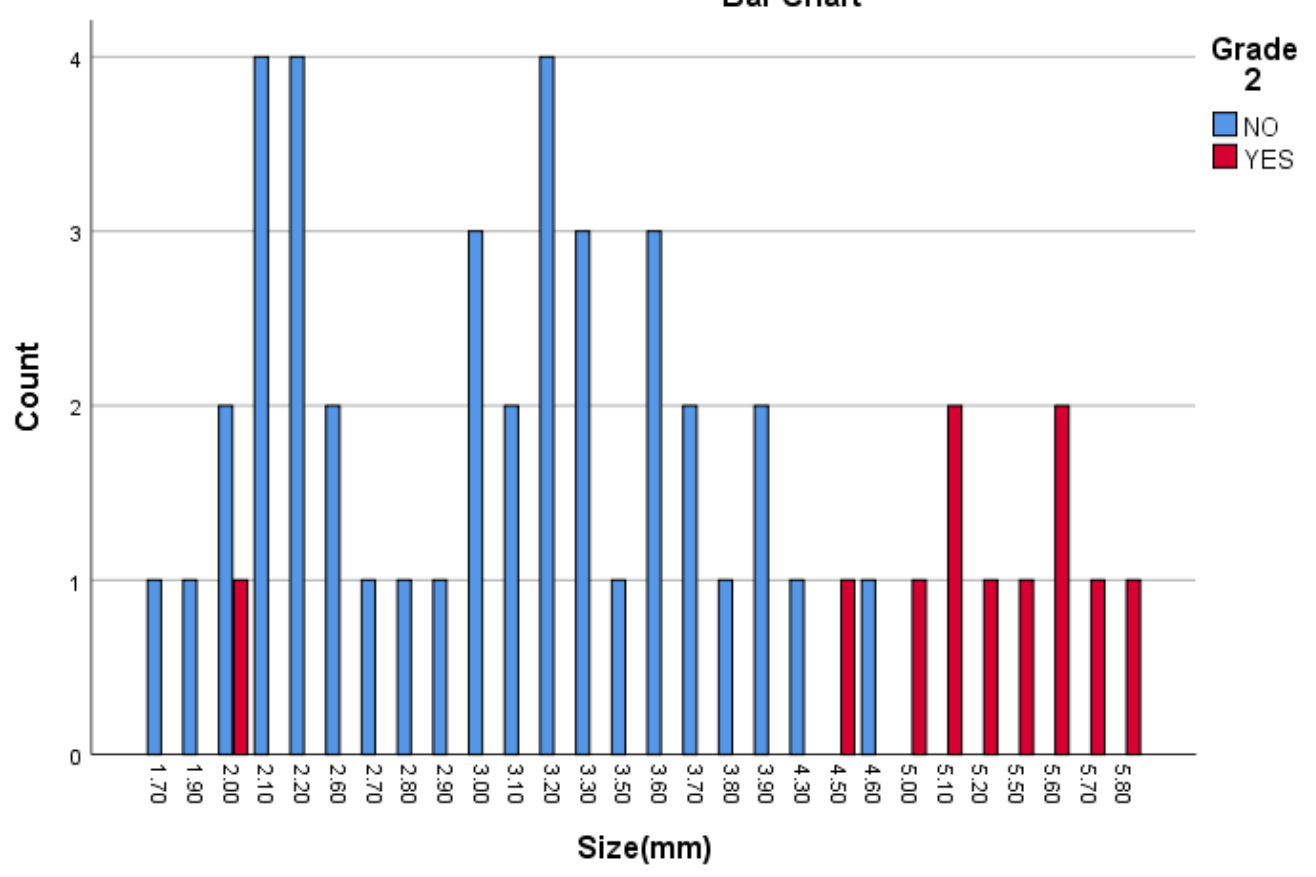

Graph 5

Table No 5.6: $\quad n=51$

\section{Size $(\mathrm{mm}) *$ Grade 3}

Count

Grade 3

\begin{tabular}{lll|l} 
& & NO & Total \\
\hline Size $(\mathrm{mm})$ & 1.70 & 1 & 1 \\
\cline { 2 - 4 } & 1.90 & 1 & 1 \\
\hline 2.00 & 3 & 3 \\
\hline 2.10 & 4 & 4 \\
\hline 2.20 & 4 & 4 \\
\hline 2.60 & 2 & 2 \\
\hline 2.70 & 1 & 1 \\
\hline 2.80 & 1 & 1 \\
\hline 2.90 & 1 & 1 \\
\hline 3.00 & 3 & 3 \\
\hline
\end{tabular}




\begin{tabular}{|c|c|c|c|}
\hline & 3.10 & 2 & 2 \\
\hline & 3.20 & 4 & 4 \\
\hline & 3.30 & 3 & 3 \\
\hline & 3.50 & 1 & 1 \\
\hline & 3.60 & 3 & 3 \\
\hline & 3.70 & 2 & 2 \\
\hline & 3.80 & 1 & 1 \\
\hline & 3.90 & 2 & 2 \\
\hline & 4.30 & 1 & 1 \\
\hline & 4.50 & 1 & 1 \\
\hline & 4.60 & 1 & 1 \\
\hline & 5.00 & 1 & 1 \\
\hline & 5.10 & 2 & 2 \\
\hline & 5.20 & 1 & 1 \\
\hline & 5.50 & 1 & 1 \\
\hline & 5.60 & 2 & 2 \\
\hline & 5.70 & 1 & 1 \\
\hline & 5.80 & 1 & 1 \\
\hline Total & & 51 & 51 \\
\hline
\end{tabular}

Table 6: This table shows the correlation between cholelithiasis size and Fatty liver grade 3.

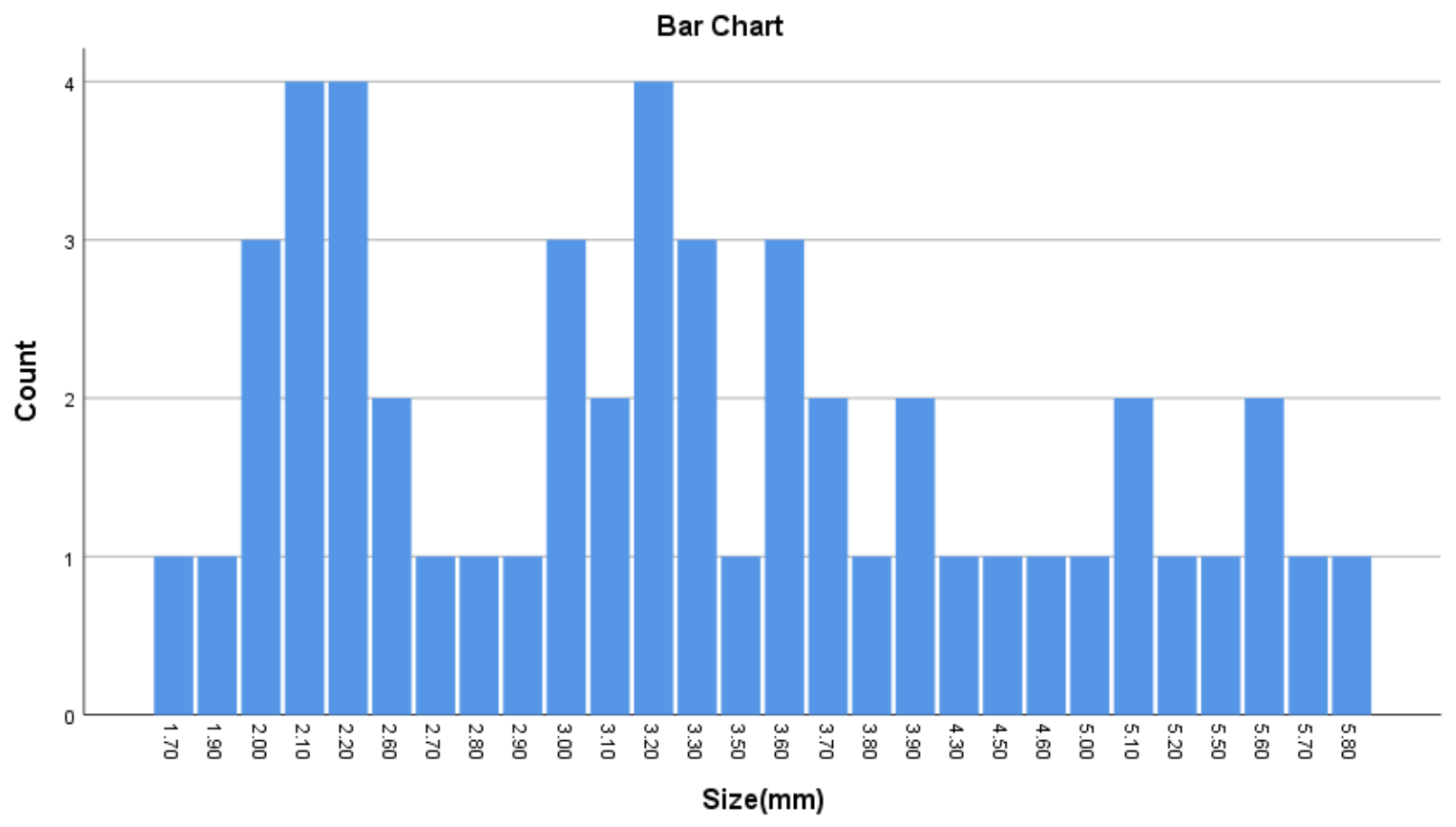

Graph 6 